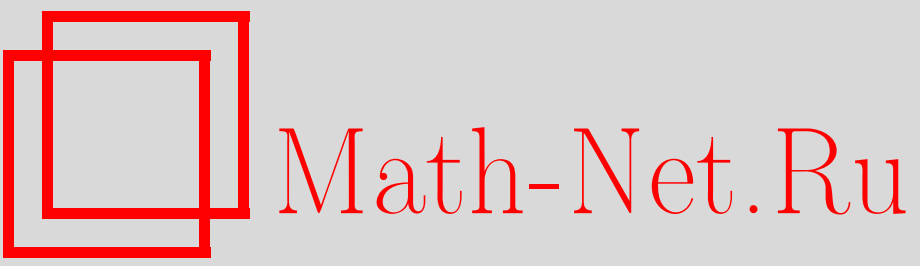

С. А. Мелихов, Р. В. Михайлов, Зацепления по модулю узлов и Проблема изотопической реализации, УМН, 2001, том 56, выпуск 2, 219-220

DOI: https://doi.org/10.4213/rm391

Использование Общероссийского математического портала Math-Net.Ru подразумевает, что вы прочитали и согласны с пользовательским соглашением

http://www.mathnet.ru/rus/agreement

Параметры загрузки:

IP : 54.80 .97 .219

26 апреля 2023 г., 10:16:24 


\title{
ЗАЦЕПЛЕНИЯ ПО МОДУЛЮ УЗЛОВ И ПРОБЛЕМА ИЗОТОПИЧЕСКОЙ РЕАЛИЗАЦИИ
}

\author{
С. А. МЕлихов, Р. В. Михайлов
}

Пусть $f$ - непрерывное отображение компактного полиэдра $X$ в многообразие $Q$. Следует ли из дискретной реализуемости, т.е. $\varepsilon$-аппроксимируемости вложениями $\forall \varepsilon>0$, отображения $f$ его изотопическая реализуемость, т.е. существование псевдоизотопии $H_{t}: Q \rightarrow Q$, $t \in I=[0,1]$ (т.е. гомотопии, при $t<1$ осуществляющей гомеоморфизм), переводящей на $f$ некоторое вложение $g: X \hookrightarrow Q$ (т.е. $H_{0}=\mathrm{id}$ и $\left.H_{1} g=f\right)$ ? Этот вопрос, восходящий к Проблеме Л.В. Келдыш (1966 г.) о реализации дико вложенных полиэдров псевдоизотопией подполиэдров, был поставлен Е. В. Щепиным в 1993 г. и известен как Проблема изотопической реализации [1]-[3]. Изначально основное внимание было приковано к случаю отображений высокой коразмерности [1], и наконец был найден контрпример [2]. В [3] построены дискретно, но не изотопически реализуемые отображения $S^{n} \rightarrow \mathbb{R}^{2 n}, n \geqslant 3$, с сингулярным множеством $\{$ точка\} $\sqcup$ ( $p$-адический соленоид), $p \geqslant 3$. Отметим, что при $m-n \geqslant 3$ дискретно реализуемое отображение $n$-полиэдра в PL- $m$-многообразие реализуемо изотопически, если оно является композицией PL-отображения и TOP-вложения [2], а при $m>\frac{3(n+1)}{2}-$ если и только если обнуляется когомологическое препятствие, лежащее, по сути, в "зазоре" между гомологиями Александрова-Чеха и Стинрода-Ситникова [2], [3], в частности, при $m=2 n+1, n>1$ [2].

Совсем иная ситуация наблюдается в классической размерности. С одной стороны, известно не реализуемое изотопически отображение $w: I \sqcup I \rightarrow \mathbb{R}^{3}$ (с образом, состоящим из двух диких дуг Уайльдера, пересекающихся в единственной точке и “локально зацепленных" в ней) [2]; по общему положению оно дискретно реализуемо. Однако неясно, насколько типичным является этот пример, в частности, можно ли в нем заменить дикие дуги на ручные, подставив вместо зацепленных пар трилистников (на дугах Уайлдера) зацепления Уайтхеда или другие зацепления с незаузленными компонентами. Более общо, существует ли локально-плоское топологическое погружение, реализуемое дискретно, но не изотопически [2; Question I]?

Назовем PL-отображение $f: S_{1}^{1} \sqcup S_{2}^{1} \rightarrow \mathbb{R}^{3} k$-квазилинком, $k=1,2, \ldots, \omega$, если для любого $i=1,2$, для любой двойной точки $p \in f\left(S_{i}^{1}\right)$ найдутся замкнутые PL-3-шары $B_{1} \subset \cdots \subset B_{k} \subset \mathbb{R}^{3} \backslash f\left(S_{3-i}^{1}\right)$ такие, что каждый $B_{j+1}$ содержит $f$-образ некоторой дуги окружности $S_{i}^{1}$, содержащей $f^{-1}\left(B_{j}\right)$, где $B_{0}=\{p\}$ (ср. с трюком Пенроуза-Уайтхеда-ЗиманаИрвина [4]). Два зацепления $S^{1} \sqcup S^{1} \hookrightarrow \mathbb{R}^{3} k$-кв азиизотопны, если они гомотопны в классе $k$-квазилинков. Очевидно, 0-квазиизотопия совпадает с гомотопией (в смысле Милнора [5]), и можно показать, что $\omega$-квазиизотопия совпадает с (не локально-плоской) PL-изотопией. Можно проверить, что инварианты Васильева (как в обычном смысле, так и в смысле [6]) порядка $\leqslant k$, инвариантные при PL-изотопии, $\bar{\mu}$-инварианты Милнора длины $\leqslant 2 k+3$ и инварианты Кохрана $\beta^{i}, i \leqslant k$, инвариантны при $k$-квазиизотопии, причем последние два неравенства точны.

Ясно, что при $k<\omega$ любые два зацепления, достаточно близкие к двум ТОР-вложениям $S^{1} \sqcup S^{1} \hookrightarrow \mathbb{R}^{3}$, гомотопным в классе вложений, $k$-квазиизотопны. Поэтому как толшко для некоторого $k<\omega$ построен инвариант $\mathscr{I}$ отношения $k$-квазиизотопии со значениями в неотрицательных целых числах $\mathbb{Z}^{+}$такой, что $\mathscr{I}\left(l_{1} \# l_{2} \# \cdots \# l_{n} \# m_{n}\right) \rightarrow \infty$ при $n \rightarrow \infty$ для некоторых зацеплений $l_{1}, l_{2}, \ldots$ с незаузленньми компонентами и произвольных $m_{1}, m_{2}, \ldots$, немедленно доказана изотопическая нереализуемость отображения $I \sqcup I \rightarrow \mathbb{R}^{3}$, составленного из $l_{1}, l_{2}, \ldots$ и тем самым являющегося локально-плоским ТОР-погружением. Возникает следующая

ПроБлема. Существует ли ненулевой инвариант $\mathscr{I}$ отношения $k$-квазиизотопии, $k<\omega$, со значениями в $\mathbb{Z}^{+}$, такой, что $\mathscr{I}(l \# m) \geqslant \mathscr{I}(l)+\mathscr{I}(m)$ для любых $l, m$ ?

При $k=\omega$ примером такого инварианта является, очевидно, $\alpha(l)$ - количество простых узлов в разложении первой компоненты $l$, гомотопически не зацепленных со второй (т.е. таких $p$,

Работа выполнена при частичной поддержке Российского фонда фундаментальных исследований (грант № 99-01-00009) и Латвийского совета по науке (грант № 01.0282). 
что эпиморфизм коммутирования $\pi_{1}\left(\mathbb{R}^{3} \backslash l\left(S_{1}^{1}\right)\right) \rightarrow \pi_{1}\left(\mathbb{R}^{3} \backslash p\left(S^{1}\right)\right)$ отправляет в 1 класс сопряженности, отвечающий второй компоненте); инвариант $\alpha$ был использован в доказательстве изотопической нереализуемости отображения $w$ [2]. С другой стороны, мы сейчас покажем, что при $k<\omega$ такой инвариант нельзя извлечь из инвариантной при $k$-квазиизотопии факторгруппы фундаментальной группы $\pi=\pi(l)=\pi_{1}\left(\mathbb{R}^{3} \backslash l\left(S^{1} \sqcup S^{1}\right)\right)$ зацепления $l$ по подгруппе $\nu_{k} \pi=\prod_{g \in \pi}[g,[g, \ldots[g, \pi] \ldots]]$ (здесь $k+2$ пары скобок), совпадающей [8; лемма 2] с подгруппой $\prod_{g \in \pi}\left[g, g_{k+1}^{\pi}\right]$, где $g_{k+1}^{\pi}=\left\langle g^{h} \mid h \in g_{k}^{\pi}\right\rangle$, и $g_{0}^{\pi}=\pi$. Точнее, это будет установлено даже для меньшей подгруппы $\widehat{\nu}_{k} \pi=\left(\nu_{k-1} a_{1}^{\pi}\right)\left(\nu_{k-1} b_{1}^{\pi}\right)$, где $a, b$ - фиксированные меридианы компонент.

Tеорема 1. $\forall k<\omega$ для любого $l$ найдется такое $l^{\prime}$, что $\pi\left(l \# l^{\prime}\right) / \widehat{\nu}_{k} \cong \mathbb{Z} * \mathbb{Z} / \widehat{\nu}_{k}$.

ДокАЗАТЕльство. В силу [8; теорема 1] факторгруппа по $\nu_{k}$ при $k<\omega$ является нильгруппой в смысле Бэра [9], значит, будучи конечнопорожденной, нильпотентна [9]. Для любого $l$ группа $\pi=\pi(l)$ порождается своими подгруппами $a_{1}^{\pi}$ и $b_{1}^{\pi}$ [5; Lemma 4], следовательно, по теореме Хирша-Плоткина факторгруппа $\pi / \widehat{\nu}_{k} \pi$ нильпотентна. Но так как связная сумма $l$ с зеркальньм зацеплением $l^{\prime}$ конкордантна тривиальному $t$, в силу теоремы Столлингса (см. [10]) нильпотентная факторгруппа $\pi\left(l \# l^{\prime}\right) / \widehat{\nu}_{k}$ изоморфна $\pi(t) / \widehat{\nu}_{k}$.

Проверяется непосредственно, что $k$-квазиизотопическим инвариантом является в действительности факторгруппа по еще меньшей подгруппе $\mu_{k} \pi=\left\langle\left[a, a^{g}\right],\left[b, b^{h}\right] \mid g \in a_{k}^{\pi}, h \in b_{k}^{\pi}\right\rangle^{\pi}$. Хотя различие между $\nu_{k}, \widehat{\nu}_{k}$ и $\mu_{k}$ весьма тонко (так, $\widehat{\nu}_{0}=\mu_{0}$, причем $\pi / \mu_{0}-$ группа Милнора $\mathscr{G}(l)$, инвариантная при гомотопии [5]), для факторгруппы по $\mu_{k}, k>0$, доказательство теоремы 1 не проходит.

Однако поскольку $\pi / \mu_{k}$ порождается $(k+2)$-энгелевыми элементами, с учетом известных результатов Р. Бэра, К. В. Грюнберга и Б. И. Плотника получается

ТЕОРема 2. Конечный или разрешимыц эпиморфный образ всякой группь $\pi(l) / \mu_{k}$, әде $k<\omega$, нильпотентен.

Тем самым из указанного эпиморфоного образа требуемьй инвариант извлечь тоже нельзя. Используя базис Холла, можно показать, что в случае 2 -порожденной $\pi$ группа $\pi / \mu_{1}$ сама нильпотентна. С помощью обобщенного инварианта Сато-Левина [6], [10] отсюда выводится

Теорема 3. Группа $\pi / \mu_{1}$ не является полным инвариантом 1-квазиизотопии.

Авторы выражают благодарность П. М. Ахметьеву и А. Ю. Ольшанскому за ценные замечания и А.С. Гертовскому, У. Кошорке, Й. Малешичу, В.М. Нежинскому, Р. Р. Садькову, Е. Г. Скляренко и А. Б. Скопенкову за стимулирующие обсуждения.

\section{СПИСОК ЛИТЕРАТУРЫ}

[1] П. М. Ахметьев // Матем. сб. 1996. T. 187. № 7. С. 3-34. [2] S. A. Melikhov. On maps with unstable singularities // Topology Appl. (to appear). [3] П. М. Ахметьев, С. А. Мелихов // Зап. науч. сем. ПОМИ. 2000. Т. 267. C. 53-87. [4] E. C. Zeeman // Topology of 3-Manifolds and Related Topics. Proceedings of the University of Georgia Institute 1961 / ed. M. K. Fort, Jr. Englewood Cliffs, NJ: Prentice-Hall, 1962. P. 187-194. [5] J. W. Milnor // Ann. of Math. 1954. V. 59. P. 177-195. [6] M. C. Irwin // Ann. Math. 1965. V. 82. P. 1-14. [7] Чан Ван Хао. // Сиб. матем. журн. 1964. T. 5. № 2. C. 459-464. [8] R. Baer // Math. Z. 1955. V. 62. № 4. P. 402-437. [9] T. D. Cochran // Math. Proc. Cambridge Philos. Soc. 1985. V. 97. P. 465-472. [10] П. М. Ахметьев, Д. Реповш // Труды МИАН. 1998. Т. 221. С. 69-80.

Московский государственный университет им. М. В. Ломоносова; Институт химической физики Латвийского университета
Принято редколлегией 01.02.2001 